\title{
Prelimbic/Infralimbic Inactivation Impairs Memory for Multiple Task Switches, But Not Flexible Selection of Familiar Tasks
}

\author{
Erin L. Rich and Matthew L. Shapiro \\ Fishberg Department of Neuroscience and Alfred B. and Gudrun J. Kastor Neurobiology of Aging Laboratories, Mount Sinai School of Medicine, New York, \\ New York 10029-6574
}

\begin{abstract}
Behavioral flexibility, in the form of strategy switching or set shifting, helps animals cope with changing contingencies in familiar environments. The prelimbic (PL) and infralimbic (IL) regions of the rat prefrontal cortex (PFC) contribute to this ability so that rats trained to use one strategy have difficulty learning a new one if the PL/IL is inactivated. Thus, the PL/IL mediates learning new tasks in place of old ones, but it may also be required to switch between familiar tasks. To test this hypothesis, we trained rats to perform multiple task switches on a plus-shaped maze, alternating between two familiar tasks. Muscimol inactivation of the PL/IL never impaired switch acquisition, but did impair memory for the recently acquired switch $24 \mathrm{~h}$ later. Additional experiments determined that control rats continued to perform the new task $24 \mathrm{~h}$ after a switch, but rats with PL/IL inactivation had impaired memory and performed the same task that was learned before inactivation. This impairment was observed in multiple switches, demonstrating that PL/IL activity was required to remember which of two familiar tasks was most recently successful. After many switches, however, muscimol no longer impaired performance, and both saline- and muscimol-infused rats appeared to use immediate task contingencies rather than memory to select among familiar tasks. This strategy may account for the decreased effect of PL/IL inactivation observed after extensive training. Thus, although PL/IL activity contributed to memory for multiple task switches, it was not required for flexibly selecting among highly familiar tasks.
\end{abstract}

Key words: prefrontal cortex; prelimbic; infralimbic; muscimol; task switch; strategy

\section{Introduction}

The PFC helps organize effective behavior and cognition (Nauta, 1971; Fuster, 1995), but the fundamental operations of the PFC, often described as "executive functions," are not well understood. The guided activation theory (Miller and Cohen, 2001) proposes that PFC neurons alter activity in other brain areas so that appropriate sensory, memory, and motor circuits are activated to guide successful behavior. Such functions are important when the organization of behavior must be modified. Indeed, damage to the human dorsolateral PFC (dlPFC) typically produces perseverative or disorganized behavior (Milner, 1982; Owen et al., 1991; Ptak and Schnider, 2004) that is evident when new or unfamiliar responses must overcome previously learned ones (Drewe, 1974; Robinson et al., 1980; Stuss et al., 2000). Thus, switching to unfamiliar responses likely engages the dlPFC. The extent to which the structure is required for switching among familiar responses is unknown. The present experiments tested PFC contributions

Received Jan. 26, 2007; revised March 2, 2007; accepted March 21, 2007.

This work was supported by National Institutes of Health Grants MH65658, MH073689, and MH077431, and the Mount Sinai School of Medicine. We thank Janina Ferbinteanu, Pam Kennedy, and Jake Young for comments on a previous draft and Anna Balk for computer programming.

Correspondence should be addressed to Dr. Matthew L. Shapiro, Mount Sinai School of Medicine, One Gustave Levy Place, Box 1065, New York, NY 10029. E-mail: Matthew.Shapiro@mssm.edu.

DOI:10.1523/JNEUROSCI.0369-07.2007

Copyright $\odot 2007$ Society for Neuroscience $\quad$ 0270-6474/07/274747-09\$15.00/0 to flexible selection between two behavioral tasks in an animal model with varied degrees of strategy familiarity.

Strategy switching paradigms have assessed PFC function in rats. Although homology of frontal regions between rats and primates is debated (Pruess, 1995), comparative anatomy and neuropsychology suggest that the rat PL/IL is homologous to the primate dIPFC (Uylings et al., 2003). Therefore, PL/ILdependent behavior in rats may provide a useful model for some aspects of human dlPFC function (Brown and Bowman, 2002). Maze tasks that must be solved by discriminating between subsets of available cues (e.g., allocentric spatial vs egocentric cues) operationally define task-solving "strategies" (e.g., "place" and "response" tasks, respectively) (Ragozzino et al., 1999b). In the place task, rats always entered the same goal arm [e.g., north (see Fig. 3)]; in the response task, rats were required to execute the same body turn (e.g., left) to enter a goal arm. Rats learned either task rapidly, even when the PL/IL was inactivated (Ragozzino et al., 1999 b). The same treatment, however, impaired task (or strategy) switching (place to response or response to place). Like patients with dlPFC lesions, rats with PL/IL inactivation perseveratively engaged previously learned tasks (Ragozzino et al., 1999a,b, 2003). In contrast, PL/IL inactivation did not impair task reversals (e.g., north to south goals or right to left turns), showing that the PL/IL was not required for detecting or responding to new reward contingencies (Ragozzino et al., 1999b, 2003). 
The present experiment aimed to clarify the cognitive processes involved in strategy switching. Although the PL/IL contributes to learning a new task that replaces an old one, task familiarity may alter this contribution. If PL/IL neurons mediate task switching per se, then their inactivation should impair switching, regardless of task familiarity. If PL/IL neurons specifically contribute to learning new tasks over old ones, inactivation should not impair switches among familiar tasks. We compared learning a novel switch to switching between two well learned tasks. For each switch, acquisition and retention were assessed independently. We found that PL/IL activity contributed to memory for task switches, even when both tasks were familiar, and suggest that the PL/IL acts to differentially bias memory systems involved in strategy switching to guide future behavior.

\section{Materials and Methods}

Animals. Thirty-four male Long-Evans rats weighing 275-325 g at the beginning of the experiment were housed individually in a colony room held on $12 \mathrm{~h}$ light/dark cycle. After acclimating to the colony room for at least $5 \mathrm{~d}$, rats were food-restricted to no $<85 \%$ of their ad libitum body weight and maintained on a restricted diet for the duration of the experiment. All procedures with animals were performed in accordance with Institutional Animal Care and Use Committee guidelines and those established by the National Institutes of Health.

Maze. An elevated plus-shaped maze, consisting of four wooden arms $(65 \times 8 \mathrm{~cm})$ meeting at $90^{\circ}$ angles was used. East and west arms were designated "start" arms and north and south arms were designated "goal" arms. Only one start arm was open on a given trial and a wooden block prevented access to the unused start arm. In each trial, both goal arms were open, but only one was baited. Food wells were drilled at the ends of all arms to hold cereal reward. The bottoms of the wells were made of mesh screen, below which an inaccessible food reward was placed to minimize the influence of odor cues in the task. A waiting platform was placed next to the maze. The maze and waiting platform were open to the testing room, which had several stationary visual cues on the walls.

Tasks. Previous experiments operationalized different strategies by using tasks that depend on different memory systems (White and McDonald, 2002). A place task and a response task required similar overt behaviors, but (see Fig. 3) depend on different memory systems, the hippocampus or dorsal striatum, respectively (White and McDonald, 2002). In both tasks, the rat was placed on a start arm and trained to approach the choice point then enter a goal arm for food reward (half of a piece of Froot Loops cereal; Kellogs, Battlecreek, MI). In the place task, rats learned to approach one of the two goal arms (north or south) from both start arms. In the response task, rats learned to make either a right or a left body turn on every trial to enter the rewarded goal arm.

Maze acclimation. Before surgery, all rats were handled and acclimated to the testing environment by allowing them to forage for food randomly scattered on the maze.

Surgery. Rats were anesthetized with continuous-flow isoflurane and mounted in a stereotaxic frame. Rectal temperature was monitored and core temperature maintained with a heating pad. The scalp was shaved, scrubbed with betadine, and incised. Burr holes were drilled at stereotaxic coordinates $+3.0 \mathrm{~mm}$ anterior and $\pm 1.8 \mathrm{~mm}$ medial/lateral from bregma. Twenty-six-gauge cannulas (Plastics-One, Roanoake, VA) were implanted at a $14^{\circ}$ angle to vertical and $-2.0 \mathrm{~mm}$ ventral to dura. The cannulas were affixed to the skull with dental acrylic and skull screws. Dust caps with dummy cannulas extending $1.5 \mathrm{~mm}$ below the cannula tips were used to maintain patent cannulas. Rats were allowed to recover for 5-10 d after surgery before beginning maze training.

Pretraining. After surgery, rats reacclimated to the maze with another foraging session. The next day, cereal reward was placed only in the food cups on both goal arms and one start arm was blocked. Rats were placed on each start arm twice and given access to both goal arms for four trials total. The direction of their first turn was recorded for each trial and three or more turns in the same direction was noted as a turning bias. If a rat displayed a turning bias and was required to learn the response task, then they were taught the response opposite to their turning bias.

Training. Each rat was pseudorandomly assigned to either the place or response task (see Fig. 3). In each trial, the rat was placed at the distal end of a start arm (east or west arm) facing the center of the maze and allowed to enter one goal arm (north or south). Entering one full body-length into a goal arm was considered a choice, but the trial did not end until the rat either proceeded to the end of the arm or attempted to turn around and enter the other arm. If the rat chose the correct arm, it was allowed to consume the food and was then placed on the waiting platform until the start of the next trial. If the rat entered the incorrect arm, then it was returned to the waiting platform with no reward. Intertrial intervals were $\sim 5-8 \mathrm{~s}$.

During training, the same start arm was used until the rat chose the correct goal arm on two consecutive trials. Then, the start arm was changed and another series of trials continued from the second start arm until the rat again entered the correct goal twice in a row, and then the start arm was changed again. Training proceeded in this manner until rats reached a training criterion of six consecutive correct trials with three start-arm reversals. Twenty-four hours after reaching this training criterion, the rats were given 24 trials with start arms selected pseudorandomly so that no more than three consecutive trials used the same start arm. During these tests, rats were required to perform at least $80 \%$ of trials correctly (four or fewer errors) each day. Rats were tested until they met this performance criterion for $2 \mathrm{~d}$.

Switch/reversal training and evaluation. Rats were assigned to perform either task switches (experiments 1-5) or spatial reversals (experiment 1 only). Twenty-four hours after reaching performance criterion in their initial task, rats in a switching group were required to switch tasks (from place to response or response to place), and rats in the reversal group performed a spatial reversal (north goal arm to south goal arm or vice versa). Both spatial reversals and response reversals are known to be PL/IL independent (Ragozzino et al., 1999b); here, we tested only serial spatial reversals to simplify the experimental design. No cues indicated that the task had changed; rather, the rats had to respond to the altered reward contingencies on the maze. Switching and reversing ability was scored in three phases.

TTC. Switch and reversal training began with the same pattern of trials as described for initial task training; that is, each start arm was used repeatedly until two consecutive trials were performed correctly, and then the start arm was switched. The number of trials required for a rat to achieve six consecutive correct trials (with three start arm reversals) defined trials to criterion (TTC).

Errors at $\mathrm{T}=0$. When a rat achieved TTC in the new task, testing continued for 24 more trials with start arms pseudorandomized as above. Errors were counted and recorded as errors at time $0(T=0)$.

Errors at $\mathrm{T}=24$. The following day, the rats were again tested for 24 trials with the same task contingencies as $24 \mathrm{~h}$ before. Errors were counted and recorded as errors at time $24 \mathrm{~h}(T=24)$. If a rat made more than four errors (performance $<80 \%$ correct), it was given the same task the next day for another session of 24 trials. Only if the rat made four or less errors was it given a second switch or reversal the next day.

$P L / I L$ inactivation. On days when infusions were delivered, either muscimol or PBS ( $0.5 \mu \mathrm{l} /$ cannula) was administered bilaterally. Muscimol (Sigma, St Louis, MO) was diluted in PBS to a concentration of 100 $\mathrm{ng} / \mu \mathrm{l}$. For all infusions, rats were briefly anesthetized with inhaled isoflurane. Dummy cannulas were removed and 33-gauge infusion cannulas extending $1.5 \mathrm{~mm}$ below the tip of the guide cannulas (final depth was $-3.5 \mathrm{~mm}$ from dura) were inserted. Infusion cannulas were connected by Silastic tubing to syringes mounted in a pump that delivered infusions at a rate of $0.25 \mu \mathrm{l} / \mathrm{min}$. Infusion cannulas were left in place for $3 \mathrm{~min}$ after injection to allow for diffusion. Rats were then placed back in their home cage to recover from the anesthesia. Twenty minutes after the infusions were completed, the rats were taken to the experimental room for testing. Behavior was also measured on days when no infusions were given to determine whether repeated muscimol infusion or isoflurane exposure altered behavior generally.

Histology. After testing was complete, rats were deeply anesthetized with isoflurane and pentobarbital $(50 \mathrm{mg} / \mathrm{ml}$, i.p. $)$ and perfused transcar- 


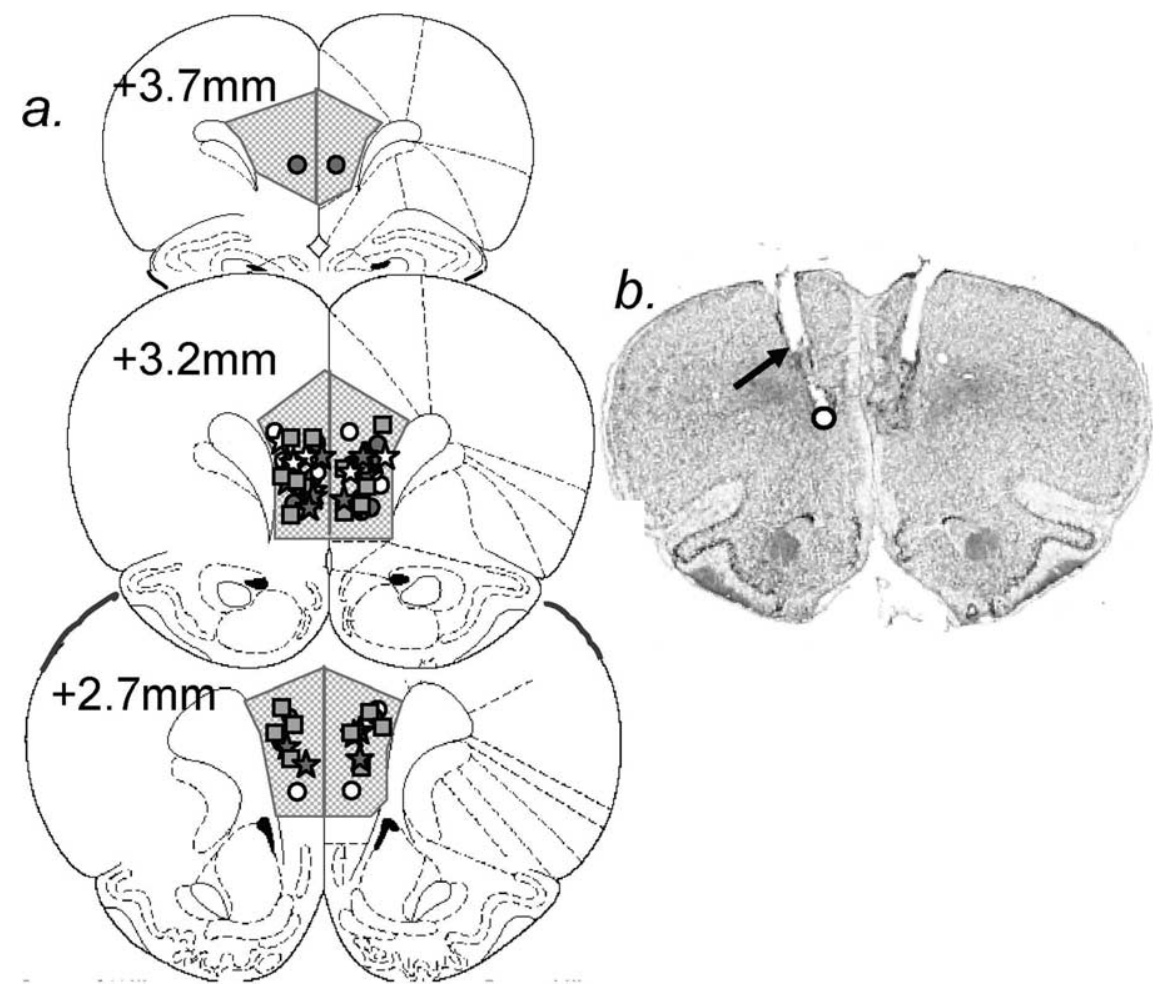

Figure 1. Cannula locations. $a$, Coronal sections +3.7 to $+2.7 \mathrm{~mm}$ from bregma are reproduced (Paxinos, 1986) with symbols indicating cannula tips for each rat. Gray circles, Muscimol infusions in the switch group of experiment 1; white circles, saline infusions in the switch group of experiment 1; gray stars, muscimol infusions in the reversal group of experiment 1; white stars, saline infusions in the reversal group of experiment 1; gray squares, experiments 3 and 4 (drug infusions were counterbalanced between rats). $\boldsymbol{b}$, A representative Nissl-stained section through the PL/IL. The black arrow indicates the end of a larger diameter track from the guide cannula, and the circle indicates the tip of the internal cannula.

dially with ice-cold PBS followed by $4 \%$ paraformaldehyde. Brains were removed and postfixed in $4 \%$ paraformaldehyde for at least $24 \mathrm{~h}$, then cryoprotected in $18 \%$ followed by $30 \%$ sucrose solution. Forty micrometer coronal sections were cut on a cryostat and mounted on slides. Sections were Nissl stained and compared with a standard brain atlas (Paxinos, 1986) to determine cannulas tip locations.

\section{Results}

\section{Histology}

In coronal sections, larger-diameter guide cannula and smallerdiameter internal cannula tracks were visible. Tips of the internal cannulas were located in either the PL or IL area, and were clustered around the PL/IL border (Fig. 1).

\section{Experiment 1: multiple switches versus multiple reversals}

To determine whether the PL/IL is required for the flexible selection and expression of familiar behaviors, 14 rats (the switch group) were trained to perform a series of 12 task switches over multiple days. To compare switching with a PL/IL-independent behavior that requires similar response flexibility (Ragozzino et al., 1999b), 10 rats (the reversal group) were trained to perform 12 spatial reversals.

Each rat in the switch group was assigned pseudorandomly to learn either response or place as an initial task. Each rat in the reversal group learned a spatial task and was assigned pseudorandomly to either the north or south goal arm as the initial rewarded location. After rats were trained in an initial task, serial switch or reversal training ensued (all training was performed as described above). TTC and errors at $T=0$ were measured on each switch or reversal day, and errors at $T=24$ were measured
24 h later. Therefore, three measurements were obtained for each of the 12 switch/ reversals (Fig. 2). If a rat performed better than $80 \%$ correct at $T=24 \mathrm{~h}$, it advanced to the next switch/reversal the following day. To assess switching among familiar tasks, each rat was trained repeatedly in two of the four possible start-goal combinations (Fig. 3). That is, if a rat learned the place task "go to the north arm" and the response task "turn left," it switched between these two familiar tasks but never learned to approach the south goal arm or to make right turns. The task combinations were counterbalanced between rats.

Each rat was randomly assigned to receive either muscimol or saline infusions and was given the same type of infusion throughout experiment 1 . Before switch or reversal numbers $1,3,6,9$, and 12 received infusions as described above (Fig. 2). No infusions were delivered during switches/ reversals $2,4,5,7,8,10$, and 11 to monitor baseline behavior in both groups. No infusions were delivered before retention trials at $T=24 \mathrm{~h}$.

To analyze learning and retention of switches and reversals, TTC and errors at $T=0$ and $T=24$ were compared separately on days when infusions were given (switch/reversal numbers 1, 3, 6, 9, and 12) using repeated-measures ANOVAs (drug by switch number). To test any persistent or nonspecific effects of muscimol, the same groups were similarly compared on days when no infusions were given (switch/reversal numbers 2, 4, 5, 7, 8, 10, and 11).

\section{Experiment 1 results: PL/IL inactivation selectively impaired} the maintenance of task switching

Muscimol-infused rats acquired task switches and initially performed the new task as well as saline-infused rats (TTC, $F_{(1,12)}=$ $3.32, p=0.094$ ) (Fig. $4 a$ ) (errors at $T=0, F_{(1,12)}=0.232, p=$ 0.639 ) (Fig. 4b). However, muscimol-infused rats made significantly more errors than saline-infused rats $24 \mathrm{~h}$ after switching (errors at $T=24$, effect of drug, $F_{(1,12)}=17.98, p=0.001$; post hoc tests, switches 1 and 3, $t_{(12)}>2.49, p<0.03$ ) (Fig. $4 c$ ). No such impairment was found in the same rats when no infusions were given (errors at $T=24, F_{(1,12)}=029, p=0.87$ ) (Fig. $4 c$ ), or in the reversal group with or without infusions (errors at $T=24$, infusions, $F_{(1,8)}=0.024 ; p=0.88$; no infusions, $F_{(6,48)}=0.59$, $p=0.74)$ (data not shown).

As rats became familiar with task switching, their performance improved. On days when infusions were delivered, rats switched in fewer trials and made fewer errors over the course of the 12 switches (TTC, $F_{(4,48)}=7.06, p<0.001$ ) (Fig. $4 a$ ) (errors at $\left.T=24, F_{(4,48)}=2.57, p=0.05\right)$ (Fig. $4 c$ ). Errors at $T=0$ were unchanged across days $\left(F_{(4,48)}=0.542 ; p=0.705\right)$ (Fig. $\left.4 b\right)$. Muscimol did not change the rate of switching improvement over days (interaction of drug by switch number, TTC, $F_{(4,48)}=$ $1.88, p=0.129$; errors at $T=0, F_{(4,48)}=1.83, p=0.139$; errors at $\left.T=24, F_{(4,48)}=1.69, p=0.168\right)$. Improved performance across days was also found when no infusions were given and in the spatial reversal group (supplemental material, available at www. 
jneurosci.org). Therefore, by the end of experiment 1 , rats became switching or reversing "experts." In addition, effects of muscimol on switch retention appeared to decrease over the course of 12 switches (Fig. 4c). Indeed, a separate ANOVA at $T=24$ in the muscimol group only showed that the effect of muscimol decreased over repeated switches $\left(F_{(4,24)}=\right.$ $2.79 ; p=0.05)$. Thus, after repeated switches, rats were less impaired by muscimol infusion at $T=24 \mathrm{~h}$ than earlier in training.

\section{Experiment 2: task switching after extensive reversal training}

Experiment 1 indicated that muscimolinduced deficits in switch retention decreased over the course of many switches. Although this decrease could be caused by familiarity with the task-switching paradigm, it could also be caused by other factors that changed over the course of the experiment (e.g., repeated intracranial infusions, increased familiarity with the maze environment, build-up of scar-tissue at the cannulas tips, etc.). To distinguish among these possibilities, rats that had performed repeated spatial reversals in experiment 1 were taught the egocentric task for the first time. These rats had cannulas for the same length of time, were given the same number of drug infusions, and were exposed the same maze environment as the task-switching rats, but had never switched between tasks.

Nine rats that had performed 12 spatial reversals were infused with muscimol $(n=4)$ or saline $(n=5)$ and taught to switch to the egocentric task as described in experiment 1 . Rats were systematically assigned to receive saline or muscimol to counterbalance previous drug exposure from experiment 1 . Testing procedures were the same as described above for task switching, and $t$ tests assessed differences between rats receiving muscimol and saline in TTC, errors at $T=0$, and errors at $T=24$.

\section{Experiment 2: results}

Memory for the new task was impaired by muscimol infusion even after extensive experience in spatial reversals, so that "reversal experts" performed identically to naive rats given muscimol before their first switch trial. As in experiment 1, switch learning and performance were the same in both saline and muscimol groups during that day $\left(\mathrm{TTC}, t_{(7)}=1.92, p=0.10\right.$; errors at $T=$ $0, t_{(7)}=1.37, p=0.21$ ) (data not shown), but rats given muscimol infusions were impaired the next day $\left(T=24, t_{(7)}=4.16\right.$, $p<0.01$ ) (Fig. 5). The results demonstrate that PL/IL function was not impaired by chronic cannulation or repeated infusion, that the brain structure remained crucial for remembering recent task switches, and that a factor specific to repeated task switching is crucial for the decreased impairment seen in experiment 1.

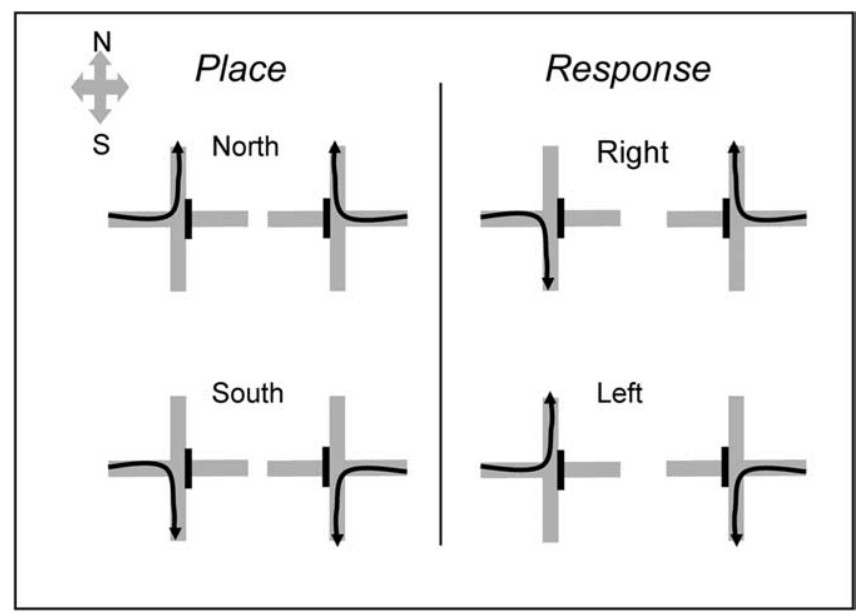

Figure 3. Place and response tasks on the plus-shaped maze. In the place task, one goal arm (north or south) was approached from both start arms (east and west). In the response task, one body turn response (right or left) was made to enter a goal arm from either start arm. Black arrows depict correct journeys from each start arm. Each rat learned only two of the four possible contingencies. Switching rats learned one place and one response contingency, whereas rats performing spatial reversals learned both place contingencies (see Experiment 1: multiple switches versus multiple reversals). 

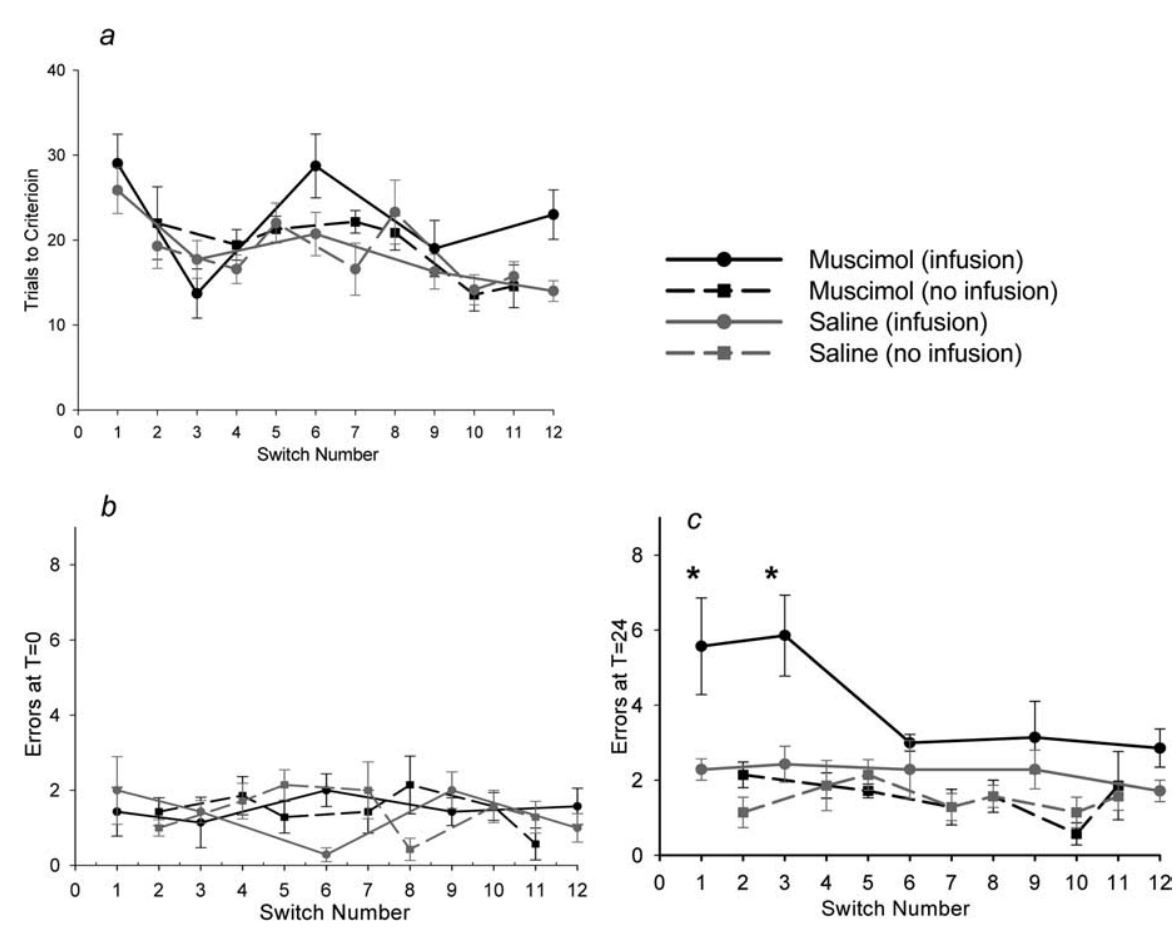

Figure 4. Experiment 1. $\boldsymbol{a}-\boldsymbol{c}$, TTC ( \pm SEM; $\boldsymbol{a})$, errors at $T=0( \pm \mathrm{SEM} ; \boldsymbol{b})$, and errors at $T=24$ ( $\pm \mathrm{SEM}$; $\boldsymbol{c}$ ) for serial task switches. Rats were infused with muscimol (black lines) or saline (gray lines) before some switches and reversals (solid lines) but not others (dashed lines). The drug infusion had no effect on any group in TTC or errors at $T=0.0$ nly rats given muscimol before switching were impaired at $T=24$ (solid black line), and this effect decreased over time. ${ }^{*} p<0.05$.

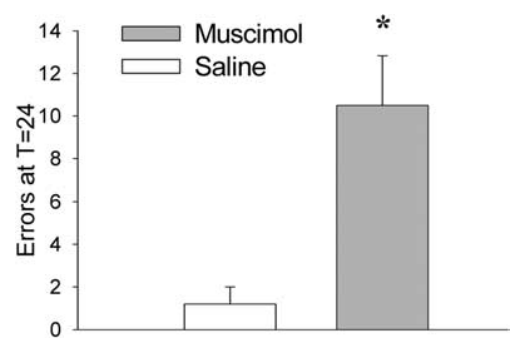

Figure 5. Experiment 2: errors at $T=24$. Errors at $T=24$ ( \pm SEM) after spatial reversal experts performed a switch are shown. Muscimol (gray) or saline (white) infusions were given before learning the switch. Muscimol significantly increased errors at $T=24$, but not TTC or errors at $T=0$ (data not shown). ${ }^{*} p<0.05$.

\section{Experiment 3: effects of muscimol given immediately after a switch}

Because muscimol infusion can reduce neuronal activity for hours (Givens and Olton, 1994; Lomber, 1999), PL/IL activity was likely suppressed both during and after switch training in experiment 1 . To determine when PL/IL activity was required for task switching, a separate group of animals was taught a task switch, but given muscimol infusion immediately after switching. Ten experimentally naive rats were housed, trained, and implanted as described above. Each rat was trained in either the place or response task, and was given either muscimol or saline infusions 3-5 min after the time 0 test (the groups were counterbalanced for both variables). Rats were returned to their home cages after infusion and given no additional testing until the following day, when they were tested at $T=24$. These data were compared with data from the first switch of the task-switching group in experiment 1 . Thus, rats were given muscimol or saline before a switch in experiment 1 and identically trained rats received muscimol or saline after a switch in experiment 3. Data were analyzed with drug by time of infusion ANOVAs and post hoc comparisons.

\section{Experiment 3: results}

Postswitching injections had no effects on subsequent behavior. Unlike rats from experiment 1 who received infusions before behavior, rats given muscimol or saline infusions immediately after performing a task switch made few errors $(T=24 \mathrm{~h}$, $t_{(8)}=0.34, p=0.74$ ) (Fig. 6). Comparing the effect of muscimol injections between the two experiments suggested that its efficacy depended on whether it was delivered before or after the switch, although the interaction did not reach statistical significance (interaction of drug by time of infusion; $\left.T=24, F_{(1,20)}=3.47, p=0.077\right)$. Post hoc comparisons confirmed that errors increased significantly only when infusions were delivered before the switch $\left(F_{(2,20)}=4.66 ; p=0.02\right)$ (Fig. 6), indicating that PL/IL activity during, but not after behavior was required for normal task switching.

\section{Experiment 4: double switch}

The data from experiments 1-3 suggest that when control rats learned a task switch, they remembered and implemented that newly learned task $24 \mathrm{~h}$ later. If muscimol impaired this memory, then rats should revert to their preswitch task $24 \mathrm{~h}$ later. In this case, rats should make fewer errors if tested on the old, preswitch task $24 \mathrm{~h}$ after muscimol infusion. In contrast, saline-infused rats should remember the most recently acquired task and therefore make more errors if tested on the old, preswitch task. We tested this hypothesis by switching the contingencies at $T=24$ back to the task performed $48 \mathrm{~h}$ previously. This testing sequence ( switch from task A to B, test $24 \mathrm{~h}$ later in task A) will be referred to as a double switch.

Rats from experiment 3 were trained in two more task switches; no infusions were given on switch 2 , but were given before switch 3, which was the double switch (Fig. 2). Therefore, the rats tested in the double-switch task had received the same type of training and had the same degree of maze and drug experience as the rats from experiment 1 during their switch 3 . This approach allowed the outcome of the double switch to be compared with standard switches in experiment 1 . In both experiments, the rats received muscimol or saline before switching, but rats from experiment 1 were tested $24 \mathrm{~h}$ later in the same task, whereas rats from experiment 3 were tested on the old task that had been performed $48 \mathrm{~h}$ before. A drug by switch type ANOVA tested for effects of drug and type of switch (switch or double switch).

\section{Experiment 4: results}

Twenty-four hours after a switch, saline-infused rats followed the task they had acquired most recently, making more errors when tested in the double-switch than in a normal switch. In contrast, muscimol-infused rats followed the task that they had performed $48 \mathrm{~h}$ before, making fewer errors in the double-switch than in the normal switch (drug by switch type, $F_{(1,20)}=15.94, p<0.001$ ) (Fig. 7a). Muscimol thus appeared to impair retention of the most recently acquired task. Improved performance in the 


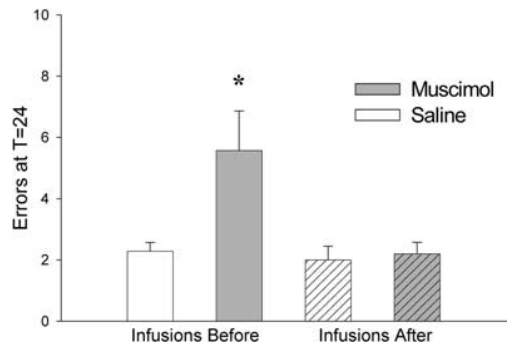

Figure 6. Experiment 3: errors at $T=24$. Errors at $T=24$ ( \pm SEM) after switching in rats infused with muscimol (gray) or saline (white) either before (solid; data from Experiment 1) or immediately after (hatched) the switch are shown. Rats were significantly impaired at $T=24$ only when infused with muscimol before switching. ${ }^{*} p<0.05$.
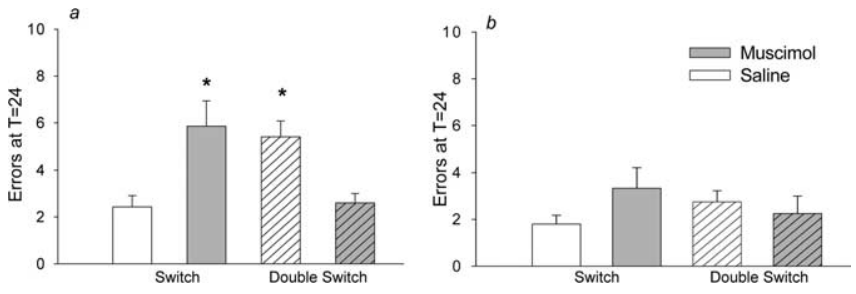

Figure 7. Experiments 4 and 5: errors at $T=24$. Errors at $T=24$ ( \pm SEM) when rats performed a switch (solid bars, data from Experiment 1) or double switch (hatched bars) are shown. Infusions of muscimol (gray) or saline (white) were given. $\boldsymbol{a}$, In experiment 4 , rats with less switching experience made more errors when infused with muscimol before switching (gray solid bars) or when infused with saline before a double switch (white hatched bars). Neither drug nor test type altered TTC or errors at $T=0$ (data not shown). $\boldsymbol{b}$, In experiment 5 , rats that were highly familiar with both tasks made few errors, regardless of infusion or switch type. ${ }^{*} p<0.05$.

double switch also demonstrated that muscimol did not cause a general increase in error rates and did not disrupt task selection in general. Rather, PL/IL inactivation caused a selective deficit in the retention of newly learned tasks. Thus, muscimol and saline infusions differentially affected performance at $T=24 \mathrm{~h}$, depending on whether rats were given a switch or a double switch. As in experiments 1-3, the acquisition of the double switch was unaffected by muscimol (TTC, $F_{(1,20)}=0.031, p=0.86$ ) errors at $T=0\left(F_{(1,20)}=0.007 ; p=0.93\right)$.

\section{Experiment 5: double switch, task switching after extensive training}

Early in training, rats given saline preferentially followed the most recently acquired task, and muscimol infusions disrupted this behavior (experiment 4). Rats given extensive switch training, however, were unimpaired by muscimol infusion and appeared to treat the task switch differently. To assess whether rats that were highly familiar with task switching relied on PL/IL to retain a switch, the same methods used in experiment 4 were used to test rats from experiment 1 in the double-switch paradigm. After switch 12, task-switching animals $(n=8)$ from experiment 1 were tested in the double-switch paradigm as described above (experiment 4). Rats were assigned to muscimol and saline cohorts that were counterbalanced for previous drug exposure and taught a 13 th switch. At $T=24 \mathrm{~h}$ they were tested on the task performed $48 \mathrm{~h}$ before (the double switch). $t$ tests compared performance between muscimol and saline groups. Drug cohorts were collapsed to compare performance on switch number 12 (experiment 1 ) to the double switch at $T=24 \mathrm{~h}$ using a paired $t$ test.

\section{Experiment 5: results}

All rats given extensive switch training performed well during the double-switch task, in contrast to rats with less switching experience. Rats infused with saline or muscimol that had performed 12 task switches performed similarly in the double switch $\left(T=24, t_{(6)}=0.56, p=0.60\right)$ (Fig. $\left.7 b\right)$, just as the same groups of rats performed similarly during the normal switch 12 (experiment 1 , errors at $T=24, t_{(6)}=1.88, p=0.11$ ). Because infusions had no effect in either the 12th (normal) or 13 th (double) switch, the drug groups were collapsed to compare performance across switch type. Regardless of whether they were tested on the new or old task at $T=24 \mathrm{~h}$, expert rats made few errors $\left(t_{(7)}=0.154 ; p=0.88\right)$, suggesting that they relied less on information from the previous day's maze experience to guide task selection. Furthermore, muscimol did not affect this flexible task selection, consistent with the idea that $\mathrm{PL} / \mathrm{IL}$ inactivation specifically disrupted the memory of the most recent switch.

\section{Discussion}

PL/IL inactivation during, but not after, learning impaired the persistence of switches when assessed $24 \mathrm{~h}$ later. In contrast, inactivation did not impair switch acquisition, performance in a new task, reversals, or switching between highly familiar tasks. The results confirm past work showing the importance of the PL/IL for efficient task switching, and further suggests that the PFC helps mediate interactions between memory systems.

\section{PL/IL inactivation impairs task switches, but not reversals} Experiment 1 verified that inactivating PL/IL impaired task switching, but not spatial reversals (Ragozzino et al., 1999a,b). Previous studies reported that PL/IL impaired switch acquisition (Ragozzino et al., 1999a,b, 2003), whereas the present results reveal an important contribution to switch maintenance (Ragozzino et al., 1999a,b). Indeed, muscimol did not affect any measure during switch acquisition (supplemental material, available at www.jneurosci.org). The following methodological factors likely explain these apparent discrepancies.

Inactivation method. Although infusion site and volume were similar, our study injected muscimol, a $\mathrm{GABA}_{\mathrm{A}}$ agonist that inactivates cell bodies, whereas other studies injected $\mathrm{Na}^{+}$channel blockers (Ragozzino et al., 1999a,b, 2003), which also inactivate fibers of passage. Whereas the muscimol infusion produced extensive inactivation of PL/IL neurons (supplemental material, available at www.jneurosci.org), $\mathrm{Na}^{+}$blockers may have produced more extensive inactivation, including disconnection, and greater functional deficits.

Measures of switch acquisition and retention. In other plusshaped maze studies, switch acquisition was measured across days and the total number of trials were combined (Ragozzino et al., 1999b). If the rats given PL/IL inactivation and trained across days acquired a switch normally within a session, but the new task was not maintained effectively across days, as we observed, then the total number of trials to criterion would be increased and acquisition would appear impaired (cf. Ragozzino et al., 1999a).

Training protocols. During switch training, rats started from the same arm repeatedly until two correct responses were made, then the start arm was changed. This procedure increased learning rates (Ragozzino et al., 1999b), perhaps by reducing interference caused by more rapid alternation of start arms. Proactive interference can alter the extent to which the PL/IL contributes to 
performance (Gisquet-Verrier and Delatour, 2006), and may differentially engage PL/IL function. Nonetheless, rats with PL/IL inactivation eventually acquire task switches (Ragozzino et al., 1999a,b), suggesting that although PL/IL activity may facilitate switching, other brain networks support learning the alternate task (albeit more slowly) without PL/IL input. The present data demonstrate that even when rats acquired and performed a switch normally, PL/IL activity influenced how the alternate task was learned, so that with normal function, the task switch was learned and remembered; with PL/IL inactivated, the task was learned, but not retained $24 \mathrm{~h}$ later.

\section{Repeated task switching alters behavioral tactics}

Performance improved over the course of 12 switches or reversals, so that both saline- and muscimol-infused rats acquired the alternate task faster and made fewer errors $24 \mathrm{~h}$ later. Muscimol effectively inactivated PL/IL even after repeated drug infusions and chronic cannulation (experiment 2), indicating that improved performance resulted from task-switching experience itself, and this experience influenced the tactical use of memory to select among familiar tasks (experiments 4 and 5). Early in training through switch 3, behavior was guided by memory for the task that was successful the day before. This memory-based approach was suggested by the good performance of control rats at $T=$ 24 in experiment 1 , and confirmed by poor performance in the double switch. At $T=24$ in the double-switch, the previous day's task (e.g., "go to the north arm") was incorrect, whereas the task from $48 \mathrm{~h}$ before (e.g., "turn right") was correct. Remembering the most recently successful task would lead to perseverative errors (going north rather than turning right). But, if memory for the previous day's task ("go north") were impaired by muscimol, while memory for the task performed $48 \mathrm{~h}$ earlier ("turn right") remained intact, then muscimol should facilitate performance at $24 \mathrm{~h}$ in the double switch. Indeed, control rats made more errors than rats given muscimol. Thus, early in training, memory for the most recently successful task guided discriminative behavior.

Later in training, however, both groups performed well in the double switch. Rather than memory, immediate task contingencies seemed to guide behavior (experiment 5). This shift may reflect the rats' learning that tasks changed frequently across days, but only among two familiar tasks. Thus, rats may have tested each of the two tasks and rapidly adopted the successful one. Such a strategy would support high performance levels whether rats were tested in a standard or double switch, precisely as observed. Alternatively, repeated switches between two tasks may produce proactive interference, so that remembering the most recent task becomes more difficult than testing each until the correct one is verified. In either case, switching flexibly between two highly familiar tasks did not require PL/IL activity, as muscimol did not impair either standard or double switches late in training. Thus, between switch 3 and 12, rats acquired a new tactic for task selection. Converging evidence from the decreased effect of PL/IL inactivation, which also occurred between switch 3 and 12, is consistent with this hypothesis. Although these experiments cannot determine causation, they suggest that this new strategy eliminated the need for PL/IL activity in the task. PL/IL inactivation only impaired behavior that was guided by memory for the most recent task switch.

PL/IL function, task switching, and multiple memory systems Converging data indicate that multiple brain systems contribute to task switching. PL/IL activity (1) is not required for acquiring or performing either the place or response task (Ragozzino et al., 1999b), (2) facilitates switch acquisition during learning (Ragozzino et al., 1999b) and persistence of that learning, and (3) is unlikely to store task memory, because previously learned tasks are unaffected by inactivation. Thus, PL/IL activity likely influences information processing and storage elsewhere. The hippocampal system is required for spatial and episodic-like memory and for performing the place task in the plus-shaped maze (O'Keefe, 1976; McDonald and White, 1993), whereas the dorsal striatal system is required for egocentric and cue-approach memory, and supports the response task (Cook and Kesner, 1988; Packard and McGaugh, 1996). The two memory systems normally function in parallel, and the plus-shaped maze can produce competition between them (White and McDonald, 2002). Indeed, hippocampal inactivation can facilitate dorsal-striatal learning (Chang and Gold, 2003). Switching, for example, from place to response should reduce the relative influence of hippocampal processing and increase that of dorsal striatal processing on ongoing behavior. PL/IL activity may contribute to this modulation.

Reciprocal connectivity between the PL/IL and both the hippocampus and the striatum could support functional modulation of memory systems in a manner analogous to Fuster's perception-action cycle (Fuster, 1995). The PL/IL receives direct afferents from the hippocampus (Jay et al., 1989; Jay and Witter, 1991; Conde et al., 1995) and innervates the hippocampal system via the nucleus reunions (Wouterlood et al., 1990; Wouterlood, 1991; Vertes, 2002; Vertes et al., 2006). The $\mathrm{PL} / \mathrm{IL}$ is also interconnected with many regions of the striatum via corticostriatal loops (Groenewegen et al., 1990; Berendse et al., 1992). The PFC is thereby well situated to modulate each system. To perform such a function, the PL/IL should integrate afferent activity from these systems with reward information. The PL/IL is innervated by dopaminergic (DA) fibers from the ventral tegmental area (Fuxe et al., 1974; Lindvall et al., 1974; Berger et al., 1976; Descarries et al., 1987), and blockade of certain DA receptors in the PL/IL has the same effect as inactivation on task switching (Ragozzino, 2002; Floresco et al., 2006). DA release can reflect both unexpected reward and the absence of expected reward (Schultz, 1997, 2006), and could thereby "gate" (O'Reilly et al., 2002) PL/IL responses to other afferents. PL/IL efferents may then alter activity in different memory systems, either directly or via modification of cortical or subcortical afferents. Such a mechanism could allow PL/IL output to correlate reward information with hippocampal and striatal input, and coordinate these systems toward optimal task performance, either inhibiting brain systems that model task contingencies poorly, increasing activity in networks that optimize task contingencies, or both.

This view suggests that without competition between brain systems, PL/IL activity would be irrelevant, for example, when learning an initial task or adapting to a new contingency using the same memory system, as in reversals. Neither initial task learning nor the acquisition or maintenance of reversals are impaired by PL/IL inactivation (Ragozzino et al., 1999a,b, 2003; Chudasama et al., 2001). Conversely, the orbitofrontal cortex (OFC) contributes to task reversals (Schoenbaum et al., 2002; McAlonan and Brown, 2003), suggesting that the OFC may bias subnetworks within systems. IL inactivation also impairs the persistence, but not the acquisition, of conditioned fear extinction after the same temporal pattern described here for task switching (Morgan et al., 1993; Quirk et al., 2000; Lebron et al., 2004; Sierra-Mercado et al., 2006). These effects are mediated by IL-enhanced inhibition of 
amygdala circuits (Milad and Quirk, 2002; Quirk et al., 2003). Thus, both extinction of conditioned fear and task switching may exemplify dynamically guided activation (Miller and Cohen, 2001), in which neuronal signals from the PL/IL integrate and modulate other brain systems to help organize behaviors that best predict task contingencies.

\section{Conclusions}

The PL/IL contributes to the maintenance of task switches, perhaps by modulating activity between memory systems. Future investigations should assess differential PL and IL contributions to switching, and probe interactions between the PL, IL, hippocampus, and striatum. This paradigm is ideal for such investigations, because the brain systems supporting place and response tasks are dissociable and relatively well understood.

\section{References}

Berendse HW, Galis-de Graaf Y, Groenewegen HJ (1992) Topographical organization and relationship with ventral striatal compartments of prefrontal corticostriatal projections in the rat. J Comp Neurol 316:314-347.

Berger B, Thierry AM, Tassin JP, Moyne MA (1976) Dopaminergic innervation of the rat prefrontal cortex: a fluorescence histochemical study. Brain Res 106:133-145.

Brown VJ, Bowman EM (2002) Rodent models of prefrontal cortical function. Trends Neurosci 25:340-343.

Chang Q, Gold PE (2003) Intra-hippocampal lidocaine injections impair acquisition of a place task and facilitate acquisition of a response task in rats. Behav Brain Res 144:19-24.

Chudasama Y, Bussey TJ, Muir JL (2001) Effects of selective thalamic and prelimbic cortex lesions on two types of visual discrimination and reversal learning. Eur J Neurosci 14:1009-1020.

Conde F, Maire-Lepoivre E, Audinat E, Crepel F (1995) Afferent connections of the medial frontal cortex of the rat. II. Cortical and subcortical afferents. J Comp Neurol 352:567-593.

Cook D, Kesner RP (1988) Caudate nucleus and memory for egocentric localization. Behav Neural Biol 49:332-343.

Descarries L, Lemay B, Doucet G, Berger B (1987) Regional and laminar density of the dopamine innervation in adult rat cerebral cortex. Neuroscience 21:807-824.

Drewe EA (1974) The effect of type and area of brain lesion on Wisconsin card sorting test performance. Cortex 10:159-170.

Floresco SB, Magyar O, Ghods-Sharifi S, Vexelman C, Tse MT (2006) Multiple dopamine receptor subtypes in the medial prefrontal cortex of the rat regulate set-shifting. Neuropsychopharmacology 31:297-309.

Fuster JM (1995) Memory in the cerebral cortex: an empirical approach to neural networks in the human and nonhuman primate. Cambridge, MA: MIT.

Fuxe K, Hokfelt T, Johansson O, Jonsson G, Lindbrink P, Ljundahl A (1974) The origin of the dopamine nerve terminals in limbic and frontal cortex. Evidence for meso-cortico dopamine neurons. Brain Res 82:349-355.

Gisquet-Verrier P, Delatour B (2006) The role of the rat prelimbic/infralimbic cortex in working memory: not involved in the short-term maintenance but in monitoring and processing functions. Neuroscience 141:585-596.

Givens B, Olton DS (1994) Local modulation of basal forebrain: effects on working and reference memory. J Neurosci 14:3578-3587.

Groenewegen HJ, Berendse HW, Wolters JG, Lohman AH (1990) The anatomical relationship of the prefrontal cortex with the striatopallidal system, the thalamus and the amygdala: evidence for a parallel organization. Prog Brain Res 85:95-116.

Jay TM, Witter MP (1991) Distribution of hippocampal CA1 and subicular efferents in the prefrontal cortex of the rat studied by means of anterograde transport of Phaseolus vulgaris-leucoagglutinin. J Comp Neurol 313:574-586.

Jay TM, Glowinski J, Thierry AM (1989) Selectivity of the hippocampal projection to the prelimbic area of the prefrontal cortex in the rat. Brain Res 505:337-340.
Lebron K, Milad MR, Quirk GJ (2004) Delayed recall of fear extinction in rats with lesions of ventral medial prefrontal cortex. Learn Mem 11:544-548.

Lindvall O, Bjorklund A, Moore R, Stenevi U (1974) Mesencephalic dopamine neurons projecting to neocortex. Brain Res 81:325-331.

Lomber SG (1999) The advantages and limitations of permanent or reversible deactivation techniques in the assessment of neural function. J Neurosci Methods 86:109-117.

McAlonan K, Brown VJ (2003) Orbital prefrontal cortex mediates reversal learning and not attentional set shifting in the rat. Behav Brain Res 146:97-103.

McDonald RJ, White NM (1993) A triple dissociation of memory systems: hippocampus, amygdala, and dorsal striatum. Behav Neurosci 107:3-22.

Milad MR, Quirk GJ (2002) Neurons in medial prefrontal cortex signal memory for fear extinction. Nature 420:70-74.

Miller EK, Cohen JD (2001) An integrative theory of prefrontal cortex function. Annu Rev Neurosci 24:167-202.

Milner B (1982) Some cognitive effects of frontal-lobe lesions in man. Philos Trans R Soc Lond B Biol Sci 298:211-226.

Morgan MA, Romanski LM, LeDoux JE (1993) Extinction of emotional learning: contribution of medial prefrontal cortex. Neurosci Lett 163:109-113.

Nauta WJ (1971) The problem of the frontal lobe: a reinterpretation. J Psychiatr Res 8:167-187.

O'Keefe J (1976) Place units in the hippocampus of the freely moving rat. Exp Neurol 51:78-109.

O’Reilly RC, Noelle DC, Braver TS, Cohen JD (2002) Prefrontal cortex and dynamic categorization tasks: representational organization and neuromodulatory control. Cereb Cortex 12:246-257.

Owen AM, Roberts AC, Polkey CE, Sahakian BJ, Robbins TW (1991) Extradimensional versus intra-dimensional set shifting performance following frontal lobe excisions, temporal lobe excisions or amygdalohippocampectomy in man. Neuropsychologia 29:993-1006.

Packard MG, McGaugh JL (1996) Inactivation of hippocampus or caudate nucleus with lidocaine differentially affects expression of place and response learning. Neurobiol Learn Mem 65:65-72.

Paxinos GW (1986) The rat brain in stereotaxic coordinates. Sydney: Academic.

Pruess TM (1995) Do rats have a prefrontal cortex? The Rose-WoolseyAkert program reconsidered. J Cogn Neurosci 7:1-24.

Ptak R, Schnider A (2004) Disorganised memory after right dorsolateral prefrontal damage. Neurocase 10:52-59.

Quirk GJ, Russo GK, Barron JL, Lebron K (2000) The role of ventromedial prefrontal cortex in the recovery of extinguished fear. J Neurosci 20:6225-6231.

Quirk GJ, Likhtik E, Pelletier JG, Pare D (2003) Stimulation of medial prefrontal cortex decreases the responsiveness of central amygdala output neurons. J Neurosci 23:8800-8807.

Ragozzino ME (2002) The effects of dopamine D(1) receptor blockade in the prelimbic-infralimbic areas on behavioral flexibility. Learn Mem 9:18-28.

Ragozzino ME, Wilcox C, Raso M, Kesner RP (1999a) Involvement of rodent prefrontal cortex subregions in strategy switching. Behav Neurosci 113:32-41.

Ragozzino ME, Detrick S, Kesner RP (1999b) Involvement of the prelimbicinfralimbic areas of the rodent prefrontal cortex in behavioral flexibility for place and response learning. J Neurosci 19:4585-4594.

Ragozzino ME, Kim J, Hassert D, Minniti N, Kiang C (2003) The contribution of the rat prelimbic-infralimbic areas to different forms of task switching. Behav Neurosci 117:1054-1065.

Robinson AL, Heaton RK, Lehman RA, Stilson DW (1980) The utility of the Wisconsin Card Sorting Test in detecting and localizing frontal lobe lesions. J Consult Clin Psychol 48:605-614.

Schoenbaum G, Nugent SL, Saddoris MP, Setlow B (2002) Orbitofrontal lesions in rats impair reversal but not acquisition of go, no-go odor discriminations. NeuroReport 13:885-890.

Schultz W (1997) Dopamine neurons and their role in reward mechanisms. Curr Opin Neurobiol 7:191-197.

Schultz W (2006) Behavioral theories and the neurophysiology of reward. Annu Rev Psychol 57:87-115.

Sierra-Mercado Jr D, Corcoran KA, Lebron-Milad K, Quirk GJ (2006) In- 
activation of the ventromedial prefrontal cortex reduces expression of conditioned fear and impairs subsequent recall of extinction. Eur J Neurosci 24:1751-1758.

Stuss DT, Levine B, Alexander MP, Hong J, Palumbo C, Hamer L, Murphy KJ, Izukawa D (2000) Wisconsin Card Sorting Test performance in patients with focal frontal and posterior brain damage: effects of lesion location and test structure on separable cognitive processes. Neuropsychologia 38:388-402.

Uylings HB, Groenewegen HJ, Kolb B (2003) Do rats have a prefrontal cortex? Behav Brain Res 146:3-17.

Vertes RP (2002) Analysis of projections from the medial prefrontal cortex to the thalamus in the rat, with emphasis on nucleus reuniens. J Comp Neurol 442:163-187.
Vertes RP, Hoover WB, Do Valle AC, Sherman A, Rodriguez JJ (2006) Efferent projections of reuniens and rhomboid nuclei of the thalamus in the rat. J Comp Neurol 499:768-796.

White NM, McDonald RJ (2002) Multiple parallel memory systems in the brain of the rat. Neurobiol Learn Mem 77:125-184.

Wouterlood FG (1991) Innervation of entorhinal principal cells by neurons of the nucleus reuniens thalami. anterograde PHA-L tracing combined with retrograde fluorescent tracing and intracellular injection with lucifer yellow in the rat. Eur J Neurosci 3:641-647.

Wouterlood FG, Saldana E, Witter MP (1990) Projection from the nucleus reuniens thalami to the hippocampal region: light and electron microscopic tracing study in the rat with the anterograde tracer phaseolus vulgaris-leucoagglutinin. J Comp Neurol 296:179-203. 